\title{
Reproductive senescence and dynamic oviposition behaviour in insects
}

\author{
MARC MANGEL ${ }^{1}$ and GEORGE E. HEIMPEL ${ }^{2 *}$ \\ ${ }^{1}$ Department of Environmental Studies, University of California, Santa Cruz, CA 95064 and ${ }^{2}$ Department of Entomology, \\ University of Wisconsin, Madison, WI 53706, USA
}

\begin{abstract}
Summary
We explore the relationship between clutch size behaviour and the likelihood of egg limitation in insects with parasitoid-like life histories. We compare the incidence of egg limitation in insects that produce clutch sizes consistent with maximizing fitness per host (i.e. the 'single host maximum', or Lack clutch size) with insects exhibiting two types of dynamic behaviour. In the first case, insects produce clutch sizes according to a rule that specifies a negative relationship between egg complement and clutch size; in the second case, clutch size is determined by both egg complement and the host encounter rate using a dynamic state-variable model. The analyses are an extension of models presented by Rosenheim (1996), which predicted a strong positive relationship between the risk of egg limitation and the host encounter rate in the absence of dynamic oviposition behaviour. Our results are consistent with Rosenheim's, but they show that dynamic behaviour can greatly reduce the risk of egg limitation and weaken the relationship between the host encounter rate and egg limitation.
\end{abstract}

Keywords: dynamic modelling; egg limitation; insect clutch size; oviposition behaviour; parasitoids; reproductive senescence

\section{Introduction}

When a female insect exhausts her egg supply (i.e. becomes egg-limited), she experiences reproductive senescence (cf. Finch, 1990). An important potential cost associated with this form of senescence is the loss of reproductive opportunity. For insects with parasitoid-like life histories (sensu Price, 1975), these opportunity costs can be manifested either by hosts that remain unutilized due to egg limitation, or by the production of low-quality offspring prior to egg limitation. This latter class of opportunity costs is important for parasitoids because host quality is typically highly variable and many parasitoids lay eggs in clutches where offspring fitness is negatively correlated with clutch size (Godfray, 1994).

Rosenheim (1996) investigated conditions under which natural selection may result in a substantial proportion of insects with parasitoid-like life histories becoming egg-limited. His work was based on a trade-off between oocyte size and number, or on a trade-off between reproduction and survival. In either case, Rosenheim's analysis suggested that the fraction of the population becoming egg-limited would be substantial and that the relationship between this fraction and host availability would be positive. However, these predictions did not take into account the possibility that host selection and oviposition behaviour might be dynamic; that is, responsive to the internal state of the insect and/or environmental conditions such as the host encounter rate.

\footnotetext{
*Address all correspondence to George E. Heimpel, Department of Entomology, University of Minnesota, St. Paul, MN 55108, USA.
} 
The risk of egg limitation has been hypothesized to be a selective agent driving the evolution of dynamic behaviour (Mangel and Ludwig, 1992; Minkenberg et al., 1992). It is therefore important to consider how dynamic behaviour itself affects egg limitation. Empirical evidence that host selection and oviposition behaviour are dynamic is accumulating from a number of insect systems (e.g. Rosenheim and Rosen, 1991; Tatar, 1991; Minkenberg et al., 1992; Collier et al., 1994; Fletcher et al., 1994; Prokopy et al., 1994; Heimpel and Rosenheim, 1995; Heimpel et al., 1996; van Randen and Roitberg, 1996). These studies document changes in behaviour with decreasing egg complement that reduce the risk of egg limitation and they provide evidence that some insects delay reproductive senescence by altering host selection and clutch size behaviour.

Here, we explore (1) the extent to which dynamic oviposition behaviour can prevent egg limitation from occurring, and (2) the effect of dynamic behaviour on the relationship between the risk of egg limitation and host availability. We begin by using Rosenheim's framework to compare egg limitation under static and dynamic host clutch size behaviour, and expand this approach by using a dynamic state-variable model to allow maximum plasticity in behaviour.

\section{Clutch size dependent upon egg complement}

Without focusing on a particular system, it is most helpful to begin with the simple characterization that clutch size decreases as egg complement decreases. Empirical support for this relationship comes from studies on the butterfly Battus philenor (Odendaal and Rausher, 1990; Tatar, 1991), the parasitoid Aphytis lingnanensis (Rosenheim and Rosen, 1991) and a number of fly species (Courtney et al., 1990; Fitt, 1990). A convenient description for the dependence of clutch size, $c$, on egg complement, $x$, is:

$$
c(x)=1+\left(\frac{x^{\gamma}}{\left(0.5 x_{\max }\right)^{\gamma}+x^{\gamma}}\right)\left(c_{\max }-1\right)
$$

In this equation, $x_{\max }$ is the maximum egg complement, $c_{\max }$ is the single host maximum clutch (e.g. Mangel, 1987; also see below) and $\gamma$ is a shape parameter. The actual clutch is the greatest integer less than $c(x)$ in Equation (1) and we do not allow clutch size to exceed the egg complement (Fig. 1).

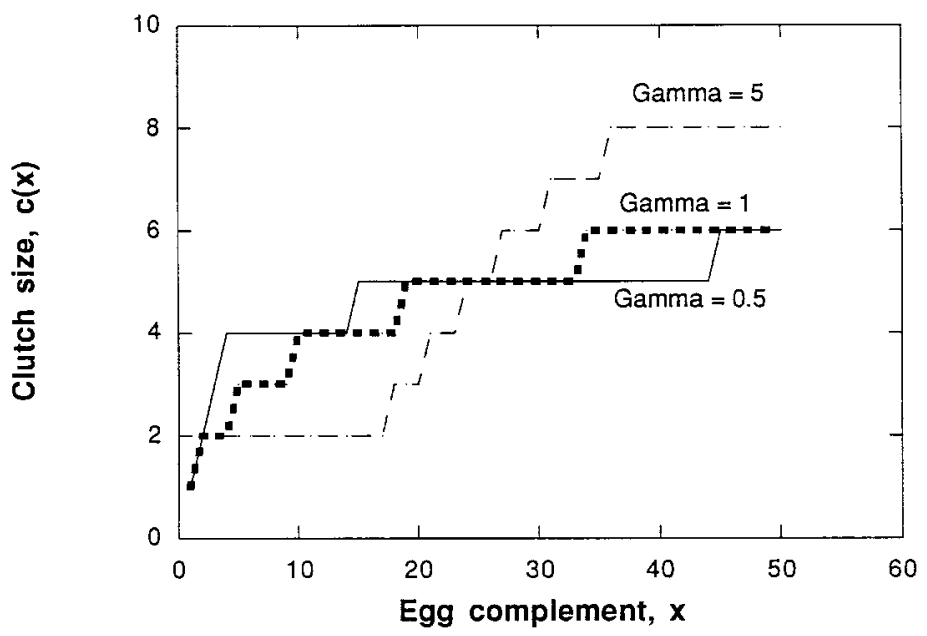

Figure 1. According to Equation (1), clutch size increases with egg complement. We show the case for which $c_{\max }=8$ and $x_{\max }=50$ for three values of the shape parameter $\gamma$ (denoted by Gamma in the figure). 
As in Rosenheim's (1996) model, we set the encounter rate to be $k$ hosts per unit time and the mortality rate to be $m$ per unit time. Thus, encountering $h$ hosts requires time $h / k$ and the parasitoid survives to this time with probability $\exp (-m h / k)$.

To compare egg limitation resulting from static and dynamic behaviour, we begin with parasitoids starting with an egg complement of $X(0)=x_{\max }$. As hosts are encountered, egg load is decremented by either $c_{\max }$, for the static case, or $c(X(t))$, for the dynamic case. Thus, the dynamics are $X(t+1)=X(t)-c_{\max }$ for the static case and $X(t+1)=X(t)-c(X(t))$ for the dynamic case, and insects are allowed to encounter hosts until $X(t)=0$. The number of hosts required to drive the egg complement to 0 is denoted by $h^{*}$, and the fraction of insects surviving to this time is $\exp \left(-m h^{*} / k\right)$. This fraction represents the proportion of individuals whose lifetime reproductive success is limited by the number of eggs available for oviposition (i.e. the fraction of egg-limited individuals). All individuals who died before laying all of their eggs can be considered time-limited.

When $c_{\max }=1$, Rosenheim's results are obtained as a limiting case (not shown). When clutch sizes can be greater than 1, the dynamic case always shows less egg limitation than the static case (Figs 2, 3). The fraction of insects becoming egg-limited is an increasing function of host availability $k$. Insects that develop on hosts that support greater egg numbers (single host maximum clutches) are more likely to experience reproductive senescence (Fig. 3).

\section{Oviposition behaviour depending upon egg complement, host encounter rate and larval density dependence}

Oviposition behaviour can also depend upon the host encounter rate itself (e.g. Strand and Godfray, 1989; Rosenheim and Rosen, 1991; Hemptinne et al., 1992; Wilson, 1994; Rosenheim and Hongkham, 1996). Rather than reparameterize Equation (1), so that clutch size also depends upon encounter rate, we solve a dynamic state-variable model (Mangel and Clark, 1988) to determine oviposition behaviour, keeping the deterministic setting. The dynamic model explicitly incorporates the host encounter rate and thus allows clutch size to vary according to both egg load and the host encounter rate.

We begin by assuming that the fitness associated with oviposition of a clutch of size $c$ in a host is

$$
f(c)=c(1-\sigma c)
$$

so that the single host maximum clutch is given by the clutch closest to $1 / 2 \sigma$ (i.e. the clutch that maximizes $f(c)$ in Equation 2 is the solution of $f^{\prime}(c)=0$ ). Larger values of $\sigma$ imply greater density dependence of larval survival within clutches (Fig. 4a). The single host maximum clutch is equivalent to the familiar 'Lack clutch size' (e.g. Godfray et al., 1991; Mangel et al., 1994), and provides the clutch size used in the static case which serves as a comparison against the dynamic case described below.

For the dynamic model, we set $F(x, t)$ to be the maximum reproductive value from time $t$ until the end of the season $T$, given that egg complement at time $t$ is $X(t)=x$ and $F(x, T)=0$. For previous times $F(x, t)$ satisfies the following dynamic programming equation (Mangel and Clark, 1988; Mangel and Ludwig, 1992):

$$
F(x, t)=\max _{c}\left\{f(c)+\mathrm{e}^{-m / k} F\left(x-c, t+\frac{1}{k}\right)\right\}
$$

The solution of this equation generates fitness, but also the optimal clutch $c^{*}(x, t)$ when egg complement is $X(t)=x$ (Fig. 4b). To determine the fraction of parasitoids experiencing egg limitation, we proceed as above, using $c^{*}(X(1), 1)$ rather than $c(x)$ from Equation (1) for the dynamic 

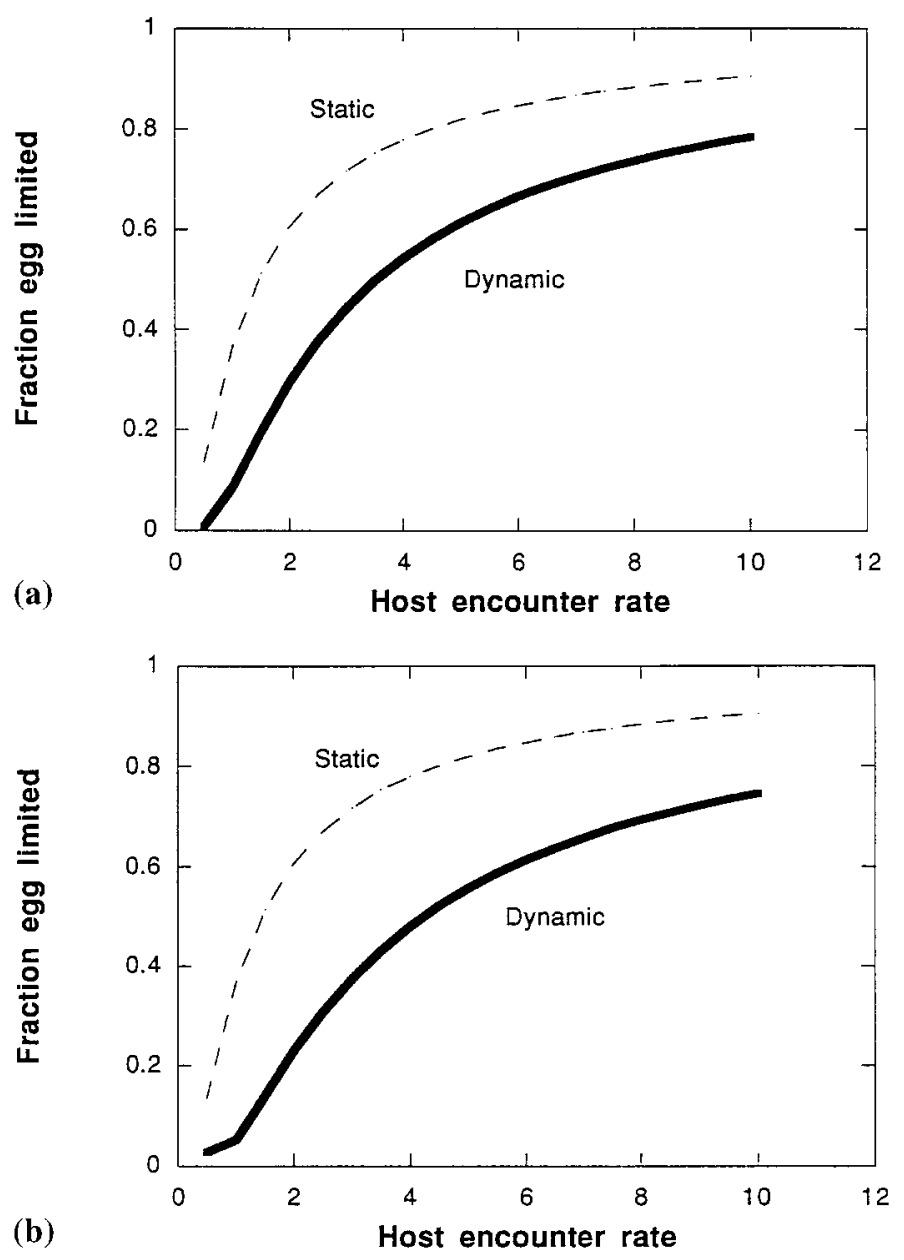

Figure 2. Dynamic oviposition behaviour, based on the general relationship shown in Fig. 1, reduces the chance of reproductive senescence. In this figure, we investigate the effects of host density and the shape parameter $\gamma$, which is 1 (a) or 10 (b). Other parameters are $x_{\max }=100, c_{\max }=5$ and $m=0.05$.

case. By using first period clutch decisions, we effectively use the stationary oviposition behaviours (Mangel and Clark, 1988). As stated above, the Lack clutch size, $1 / 2 \sigma$, was used for the static case.

The results (Fig. 5) are similar to the previous analysis. However, we now predict that increased larval density dependence will decrease the likelihood of adult reproductive senescence.

\section{Discussion}

Rosenheim (1996) considered whether egg limitation could be a plausible outcome of the evolutionary process and concluded that indeed it could be, because natural selection cannot produce perfect matching between egg production and variation in reproductive opportunity. Here, we have used the paradigm of clutch size in insects to show that including dynamic behaviour confirms Rosenheim's general conclusions, but also decreases the likelihood of egg limitation. Furthermore, 

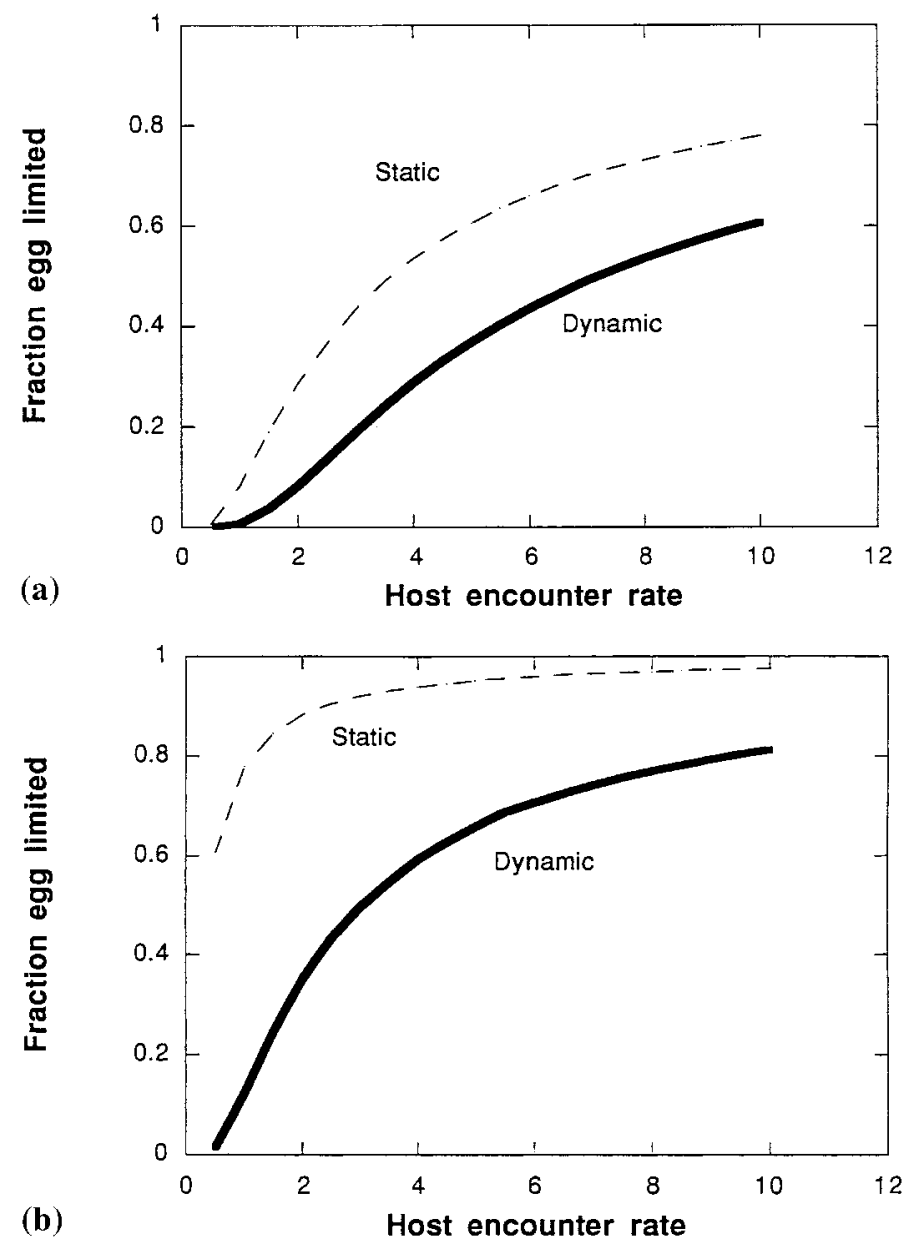

Figure 3. Effects of host density and maximum clutch size $c_{\max }$ on the fraction of insects that are egg-limited. (a) $c_{\max }=2$; (b) $c_{\max }=20$. Other parameters are $x_{\max }=100, \gamma=10$ and $m=0.05$.

we predict that egg limitation will be more likely if larval density dependence is low when this leads to larger clutches being laid. This observation is in congruence with J.T. Bonner's emphasis of selection on the life cycle, rather than any single part of it (e.g. Bonner, 1993). For instance, there is considerable controversy about the adaptiveness of female reproductive senescence (menopause) in humans (Austad, 1994). Our work suggests that reproductive senescence may be the by-product of adaptive behaviour and variation in reproductive opportunity. By itself, then, reproductive senescence may be neither adaptive nor non-adaptive when considered in the context of the life cycle.

Our analyses have shown that one example of dynamic behaviour - namely, the dependence of clutch size on egg load and the host encounter rate - allows insects to delay reproductive senescence. This buffering effect can lead to striking decreases in the proportion of a population expected to become egg-limited. Also, as might be expected, the difference between static and dynamic models is greatest when maximum clutch size is large. This reflects the greater range in clutch sizes available to insects exhibiting dynamic behaviour and was observed when maximum clutch size was 

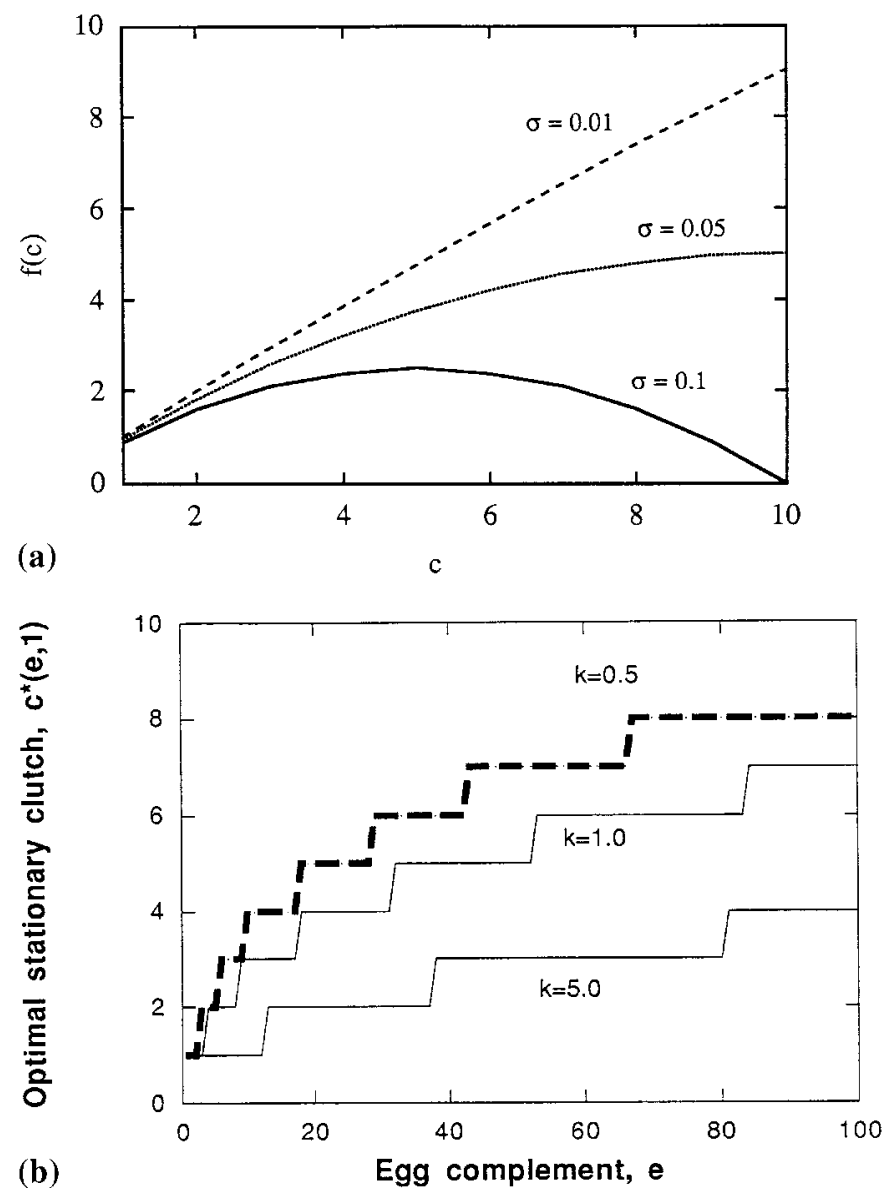

Figure 4. (a) Effect of clutch size, $c$, on fitness payoff, $f(c)$, as described by Equation (2) for three values of $\sigma$. (b) When $\sigma=0.05$, the single host maximum clutch size is 10 , but this is never achieved by the solution of the dynamic state-variable model. We show optimal first period clutches $c^{*}(\mathrm{e}, 1)$ for three values of the host encounter rate, $k$.

set explicitly or when maximum clutch size was an indirect consequence of levels of larval density dependence.

The twin observations from the empirical literature that (1) complete egg exhaustion occurs in the field (Odendaal, 1989; Driessen and Hemerik, 1992; Heimpel and Rosenheim, 1998), and (2) dynamic host selection and clutch size behaviour has been documented in the laboratory (e.g. Rosenheim and Rosen, 1991; Collier et al., 1994; Fletcher et al., 1994; Heimpel and Rosenheim, 1995; van Randen and Roitberg, 1996), in field cages (Prokopy et al., 1994) and in insects foraging freely in the field (Odendaal and Rausher, 1990; Tatar, 1991; Heimpel et al., 1996), suggest that the extent of egg limitation observed in the field reflects a risk of egg limitation that is attenuated by dynamic behaviour. However, comparing our predictions with those of Rosenheim (1996) is difficult due to the paucity of more detailed field data. Very few studies have attempted to characterize the extent of egg limitation in field populations of insects (Heimpel and Rosenheim, 1998), and even fewer studies have correlated egg limitation to host encounter rates in the field. 

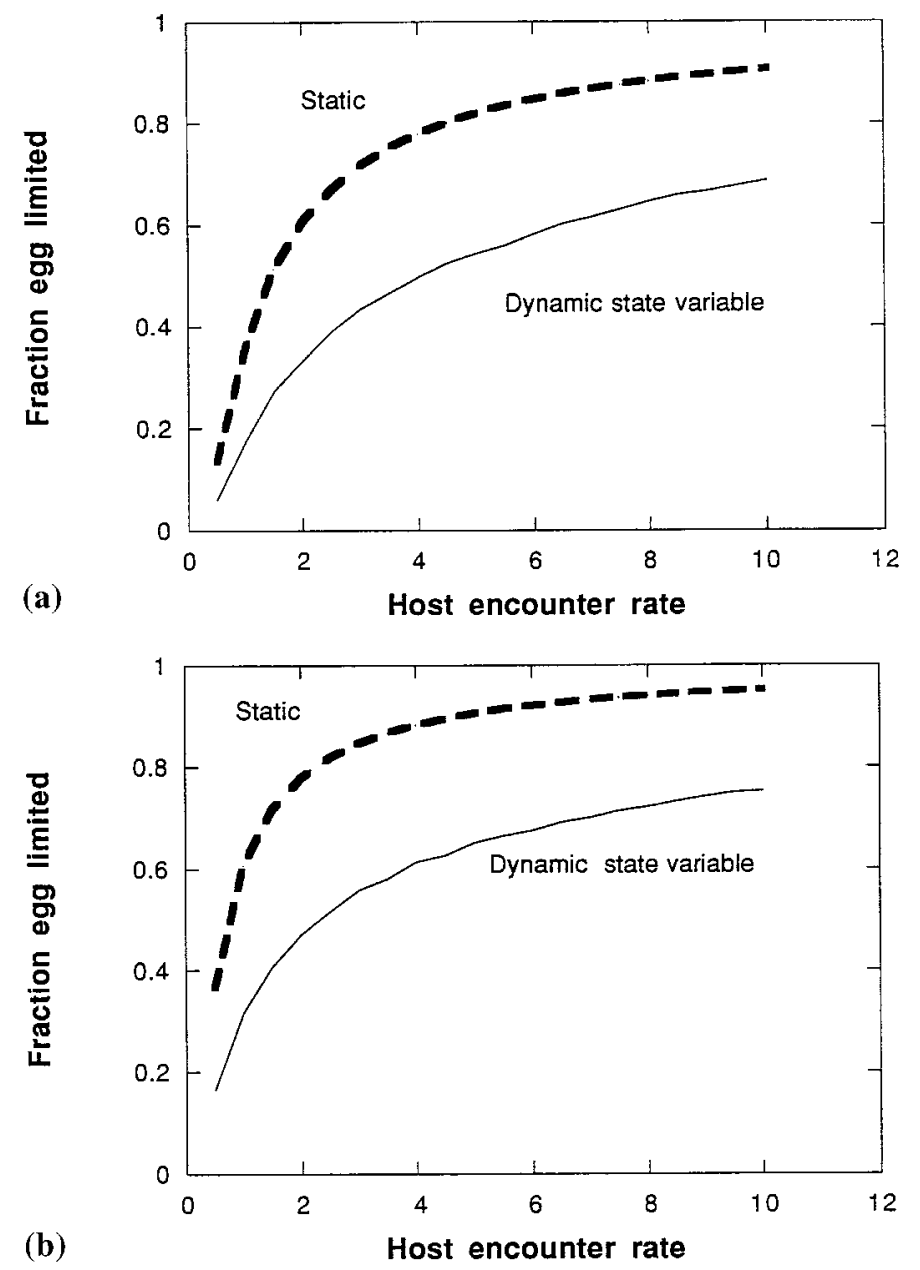

Figure 5. Similar results are obtained when trade-offs in oviposition behaviour are predicted on the basis of a dynamic state-variable model (based on the solution of Equation 3). In both cases, the parameters are $m=0.05, T=300$ and $x_{\max }=100$. For (a) $\sigma=0.1$ (single host maximum clutch size $=5$ ) and for (b) $\sigma=0.05$ ( single host maximum clutch size $=10$ ).

Both the models presented here and those of Rosenheim (1996) predict that the risk of egg limitation should increase with the host encounter rate, a prediction which has been made by others in various contexts (e.g. Morrison and Strong, 1981; Lessells, 1985; Stiling, 1987; Walde and Murdoch, 1988; van Dijken et al., 1991; Driessen and Hemerik, 1992; Hunter and Godfray, 1995). To our knowledge, the only study explicitly attempting to relate egg limitation to the host encounter rate in the field was done on the parasitoid Aphytis aonidiae (Heimpel and Rosenheim, 1998). In this study, no relationship was found between the number of mature eggs carried by foraging females ('egg load') and the density of hosts. Heimpel and Rosenheim argued previously that this unexpected result could potentially be explained by dynamic oviposition behaviour. Our analyses here, however, cast doubt upon this interpretation, as do a set of simulations parame- 
terized for the A. aonidiae system (Heimpel et al., in press; G.E. Heimpel, unpublished simulations). Although we have shown here that dynamic behaviour can weaken the relationship between egg limitation and host encounter rate, we have not shown that this relationship can be eliminated altogether.

\section{Acknowledgements}

We thank Jay Rosenheim for comments on the manuscript. This work is dedicated to John Bonner, with respect and admiration.

\section{References}

Austad, S.N. (1994) Menopause: An evolutionary perspective. Exp. Geront. 29, 255-263.

Bonner, J.T. (1993) Life Cycles. Princeton University Press, Princeton, NJ.

Collier, T.R., Murdoch, W.W. and Nisbet, R.M. (1994) Egg load and the decision to host feed in the parasitoid Aphytis melinus. J. Anim. Ecol. 63, 299-306.

Courtney, S.P., Kibota, T.T. and Singleton, T.A. (1990) Ecology of mushroom-feeding Drosophila. Adv. Ecol. Res. 20, 225-274.

Driessen, G. and Hemerik, L. (1992) The time and egg budget of Leptopilina clavipes, a parasitoid of larval Drosophila. Ecol. Ent. 17, 17-27.

Finch, C.E. (1990) Longevity, Senescence and the Genome. University of Chicago Press, Chicago, IL.

Fitt, G.P. (1990) Comparative fecundity, clutch size, ovariole number and egg size of Dacus tryoni and D. jarvisi (Diptera: Tephritidae), and their relationship to body size. Ent. Exp. Appl. 55, 11-21.

Fletcher, J.P., Hughes, J.P. and Harvey, I.F. (1994) Life expectancy and egg load affect oviposition decisions of a solitary parasitoid. Proc. Roy. Soc. Lond. B 258, 163-167.

Godfray, H.C.J. (1994) Parasitoids: Behavioural and Evolutionary Ecology. Princeton University Press, Princeton, NJ.

Godfray, H.C.J., Partridge, L. and Harvey, P.H. (1991) Clutch size. Annu. Rev. Ecol. Syst. 22, 409-429.

Heimpel, G.E. and Rosenheim, J.A. (1995) Dynamic host feeding by the parasitoid Aphytis melinus: The balance between current and future reproduction. J. Anim. Ecol. 64, 153-167.

Heimpel, G.E. and Rosenheim, J.A. (1998) Egg limitation in parasitoids: A review of the evidence and a case study. Biological Control 11, 160-168.

Heimpel, G.E., Rosenheim, J.A. and Mangel, M. (1996) Egg limitation, host quality, and dynamic behaviour by a parasitoid in the field. Ecology 77, 2410-2420.

Heimpel, G.E., Mangel, M. and Rosenheim, J.A. (in press) Effects of egg- and time-limitation on lifetime reproductive success of a parasitoid in the field. Am. Nat.

Hemptinne, J.-L., Dixon, A.F.G. and Coffin, J. (1992) Attack strategy of ladybird beetles (Coccinellidae): Factors shaping their numerical response. Oecologia 90, 238-245.

Hunter, M.S. and Godfray, H.C.J. (1995) Ecological determinants of sex allocation in an autoparasitoid wasp. J. Anim. Ecol. 64, 95-106.

Lessells, C.M. (1985) Parasitoid foraging: Should parasitism be density dependent? J. Anim. Ecol. 54, $27-41$.

Mangel, M. (1987) Oviposition site selection and clutch size in insects. J. Math. Biol. 25, 1-22.

Mangel, M. and Clark, C.W. (1988) Dynamic Modeling in Behavioural Ecology. Princeton University Press, Princeton, NJ.

Mangel, M. and Ludwig, D. (1992) Definition and evaluation of behavioural and developmental programs. Annu. Rev. Ecol. Syst. 23, 507-536.

Mangel, M., Rosenheim, J.A. and Adler, F.R. (1994) Clutch size, offspring performance, and intergenerational fitness. Behav. Ecol. 5, 412-417.

Minkenberg, O.P.J.M., Tatar, M. and Rosenheim, J.A. (1992) Egg load as a major source of variability in insect foraging and oviposition behaviour. Oikos 65, 134-142.

Morrison, G. and Strong, D.R. (1981) Spatial variation in egg density and the intensity of parasitism in a neotropical chrysomelid (Cephaloleia consanguinea). Ecol. Ent. 6, 55-61. 
Odendaal, F.J. (1989) Mature egg number influences the behaviour of female Battus philenor butterflies. $J$. Insect Behav. 2, 15-25.

Odendaal, F.J. and Rausher, M.D. (1990) Egg load influences search intensity, host selectivity, and clutch size in Battus philenor butterflies. J. Insect Behav. 3, 183-193.

Price, P.W. (1975) Reproductive strategies of parasitoids. In Evolutionary Strategies of Parasitoids (P.W. Price, ed.), pp. 87-111. Plenum Press, New York.

Prokopy, R.J., Roitberg, B.D. and Vargas, R.I. (1994) Effects of egg load on finding and acceptance of host fruit in Ceratitis capitata flies. Physiol. Ent. 19, 124-132.

Rosenheim, J.A. (1996) An evolutionary argument for egg limitation. Evolution 50, 2089-2094.

Rosenheim, J.A. and Hongkham, D. (1996) Clutch size in an obligately siblicidal parasitoid wasp. Anim. Behav. 51, 841-852.

Rosenheim, J.A. and Rosen, D. (1991) Foraging and oviposition decisions in the parasitoid Aphytis lingnanensis: Distinguishing the influences of egg load and experience. J. Anim. Ecol. 60, 873-893.

Stiling, P.D. (1987) The frequency of density dependence in insect host-parasitoid systems. Ecology 68, 844 856.

Strand, M.R. and Godfray, H.C.J. (1989) Superparasitism and ovicide in parasitic Hymenoptera: A case study of the ectoparasitoid Bracon hebetor. Behav. Ecol. Sociobiol. 24, 421-432.

Tatar, M. (1991) Clutch size in the swallowtail butterfly, Battus philenor: The role of host quality and egg load within and among seasonal flights in California. Behav. Ecol. Sociobiol. 28, 337-344.

van Dijken, M.J., Neuenschwander, P., van Alphen, J.J.M. and Hammond, W.N.O. (1991) Sex ratios in field populations of Epidinocarsis lopezi, an exotic parasitoid of the cassava mealybug, in Africa. Ecol. Ent. 16, 233-240.

van Randen, E.J. and Roitberg, B.D. (1996) The effect of egg load on superparasitism by the snowberry fly. Ent. Exp. Appl. 79, 241-245.

Walde, S.J. and Murdoch, W.W. (1988) Spatial density dependence in parasitoids. Annu. Rev. Entomol. 33, 441-466.

Wilson, K. (1994) Evolution of clutch size in insects. II. A test of static optimality models using the beetle Callosobruchus maculatus (Coleoptera: Bruchidae) J. Evol. Biol. 7, 365-386. 